\title{
Bilateral acute iris transillumination following systemic administration of antibiotics
}

\author{
Paris Tranos ${ }^{1} \cdot$ Evangelos Lokovitis $^{2} \cdot$ Stelios Masselos $^{3} \cdot$ Nikolaos Kozeis $^{1} \cdot$ Magda Triantafylla $^{1}$. \\ Nikolaos Markomichelakis ${ }^{3}$
}

Received: 27 May 2017 / Revised: 14 January 2018 / Accepted: 29 January 2018 / Published online: 2 March 2018

(c) The Royal College of Ophthalmologists 2018

\begin{abstract}
Objectives To describe the demographic characteristics, clinical features, and potential prognostic factors of bilateral acute iris transillumination (BAIT) following oral antibiotic uptake.

Methods A retrospective study of 16 consecutive patients who developed BAIT following treatment with systemic antibiotics. Detailed past medical and ocular history was obtained, presenting signs and symptoms were documented and demographic characteristics were analyzed. All patients underwent a complete ocular examination and laboratory investigation. The course of best corrected visual acuity (BCVA), anterior chamber activity, and intraocular pressure (IOP) during the follow-up period were recorded and possible correlations with potential prognosticators were investigated.

Results Fourteen females and two males were included in the present study. The mean age (SD) of the patients was 43 (14) years. All individuals presented conjunctival injection and photophobia and developed bilateral transillumination defects, fixed mid-dilated pupils and pigment dispersion in the anterior chamber. Systemic antibiotics were previously prescribed in all cases (13 patients with moxifloxacin and three patients with clarithromycin) and the mean (SD) interval between onset of symptoms and antibiotic administration was 17 (4) days. Ocular hypertension complicated all eyes and required antiglaucoma medication in 25 eyes. Severe anterior chamber pigment dispersion and higher IOP during the first week after presentation was significantly associated with longer duration of ocular hypertension (OHT) $(p=0.019)$.

Conclusions BAIT represents a rare clinical entity with characteristic features. Although etiopathogenesis of this condition remains unclear, a series of cases that indicate a strong correlation between systemic antibiotic administration and BAIT is herein presented.
\end{abstract}

\section{Introduction}

Bilateral acute iris transilumination (BAIT) is a newly recognized condition characterized by bilateral diffuse transilumination defects of the iris, pigment showering, persistent mydriasis of the pupil and occasional ocular hypertension [1]. Other conditions which are also associated

Evangelos Lokovitis

elokovitis@hotmail.com

1 Ophthalmica Eye Institute, Vitreoretinal and Uveitis department, Thessaloniki, Greece

2 Northampton General Hospital, Ophthalmology department, Northampton, UK

3 Institute of Ocular Inflammation and Pathology of the Eye, Athens, Greece with iris atrophy include pigment dispersion syndrome (PDS), Fuchs uveitic syndrome, herpetic iridocyclitis, pseudoexfoliation syndrome, Vogt-Koyanagi-Harada disease, and acute angle closure and trauma [2-5].

Many authors [6-10], implicate the systemic use of fluoroquinolones and especially moxifloxacin as the main causative factor of BAIT, while others [1], believe that a viral infection may be the triggering factor of this clinical entity. We report the clinical characteristics and course in 16 patients who developed BAIT following systemic use of moxifloxacin and clarithromycin.

\section{Materials and methods}

Sixteen consecutive patients who presented at two different ophthalmology clinics in Greece with BAIT following oral moxifloxacin or clarithromycin administration were 
included in our study. The study was conducted according to the tenets of the Declaration of Helsinki and was approved by the Ophthalmica Eye Institute ethics committee. A thorough past ocular and medical history was obtained by all patients. During each visit, the patients underwent a comprehensive ophthalmic examination that included best corrected visual acuity (BCVA), slit-lamp biomicroscopy, Goldman applanation tonometry, gonioscopy and fundus examination with indirect ophthalmoscopy. The BCVA was calculated using a Snellen scale with decimal notation and SUN grading system was used to document inflammatory and pigment activity in the anterior chamber (AC).

Routine laboratory work-up included erythrocyte sedimentation rate, complete blood count, liver enzymes, blood urea nitrogen test, creatinine, and serum serology for Varicella-Zoster virus (VZV), Cytomegalovirus (CMV), Epstein Barr Virus (EBV), and Herpes simplex virus (HSV). Anterior chamber tap for polymerase chain reaction (PCR) to investigate the presence of HSV, VZV, CMV, was performed in six patients while anterior segment optical coherence tomography (AS-OCT) was performed in four patients.

The main objectives were to evaluate the demographic features and the clinical course of the condition, including initial and late ocular signs, as well as BCVA and intraocular pressure (IOP) changes from baseline to the final visit. Underlying illnesses and types of systemic antibiotic used before the onset of symptoms were also documented. Possible correlations between IOP, demographic data, medications used and clinical signs were investigated. Snellen visual acuity was converted to logarithm of the minimum angle of resolution for the statistical analysis. Box and whisker plots and histograms were used to summarize distributions. Relationships of certain complications with baseline characteristics and possible explanatory variables were evaluated with parametric methods including independent $t$-test for normally distributed variables. Nonparametric tests, including Mann-Whitney U- and Spearman rank correlation test, were used for variables which showed non-normality. Relationships between categorical variables were evaluated using $\chi^{2}$-test. All tests of association were considered to be statistically significant if $P<$ 0.05. Analyses were performed using Microsoft Excel 2007 for Windows (Microsoft Corporation, Redmond, WA) and SPSS version 16.0 for Windows (SPSS, Inc, Chicago, IL).

\section{Results}

The mean age (SD) of the patients was 43 (14) years (range: 22-86 years), with females largely outnumbering males (14:2, respectively). In all cases there was previous
Table 1 Demographic and clinical characteristics of patients with BAIT

\begin{tabular}{|c|c|c|}
\hline \multirow{2}{*}{$\frac{\text { Characteristics }}{\text { Mean age, years (SD) }}$} & \multicolumn{2}{|l|}{ Values } \\
\hline & 43 & $(14)$ \\
\hline \multicolumn{3}{|l|}{ Gender } \\
\hline Male & 2 & $13 \%$ \\
\hline Female & 14 & $87 \%$ \\
\hline \multicolumn{3}{|l|}{ Underlying disease, no. of patients, $\%$} \\
\hline Pneumonia & 8 & $50 \%$ \\
\hline Sinusitis & 2 & $13 \%$ \\
\hline Keratitis & 1 & $6 \%$ \\
\hline Urethritis & 1 & $6 \%$ \\
\hline Appendicectomy & 1 & $6 \%$ \\
\hline Tonsilitis & 1 & $6 \%$ \\
\hline Bronchitis & 1 & $6 \%$ \\
\hline Nephrectomy & 1 & $6 \%$ \\
\hline \multicolumn{3}{|l|}{ Systemic antibiotic used, no. of patients, $\%$} \\
\hline Moxifloxacin & 13 & $81 \%$ \\
\hline Clarithromycin & 3 & $19 \%$ \\
\hline $\begin{array}{l}\text { Interval between antibiotic uptake and onset of } \\
\text { symptoms, days (SD) }\end{array}$ & 17 & (4) \\
\hline Mean follow-up time, days (SD) & 129 & $(38)$ \\
\hline Ocular hypertension, no. of eyes, $\%$ & 32 & $100 \%$ \\
\hline Posterior synechiae, no. of eyes, $\%$ & 4 & $25 \%$ \\
\hline Mean duration of topical steroids, weeks (SD) & 4.0 & $(1.78)$ \\
\hline \multicolumn{3}{|l|}{ Mean LogMAR best corrected visual acuity (SD) } \\
\hline Baseline & 0.0181 & $(0.5)$ \\
\hline Final & 0.0029 & $(0.11)$ \\
\hline \multicolumn{3}{|l|}{ Mean IOP, $\mathrm{mmHg}(S D)$} \\
\hline Baseline & 21 & $(9.9)$ \\
\hline Final & 17 & $(3.4)$ \\
\hline \multicolumn{3}{|l|}{ Lens status, no. of eyes $\%$} \\
\hline Phakic & 32 & $100 \%$ \\
\hline Aphakic, Pseudophakic & 0 & $0 \%$ \\
\hline Distorted pupillary borders, no. of eyes, $\%$ & 20 & $60 \%$ \\
\hline Diffuse Iris transillumination, no. of eyes, $\%$ & 32 & $100 \%$ \\
\hline Atonic pupil with sphincter paralysis, no. of eyes, $\%$ & 32 & $100 \%$ \\
\hline
\end{tabular}

administration of systemic moxifloxacin (13 patients) or clarithromycin (3 patients). Eleven patients claimed to be first-time users. However, five patients were not confident to report that they were treatment naive for the abovementioned medications. In 8 patients $(50 \%)$ the underlying cause for oral administration of antibiotics was pneumonia. The rest of the cases included two cases of sinusitis and individual cases of keratitis, urethritis, tonsillitis, bronchitis, nephrectomy, and appendicectomy. The mean interval between the first day of antibiotic uptake and the onset of ocular symptoms was 17 days (range: 10-25 days, SD: 4 days) and the mean follow-up time was 


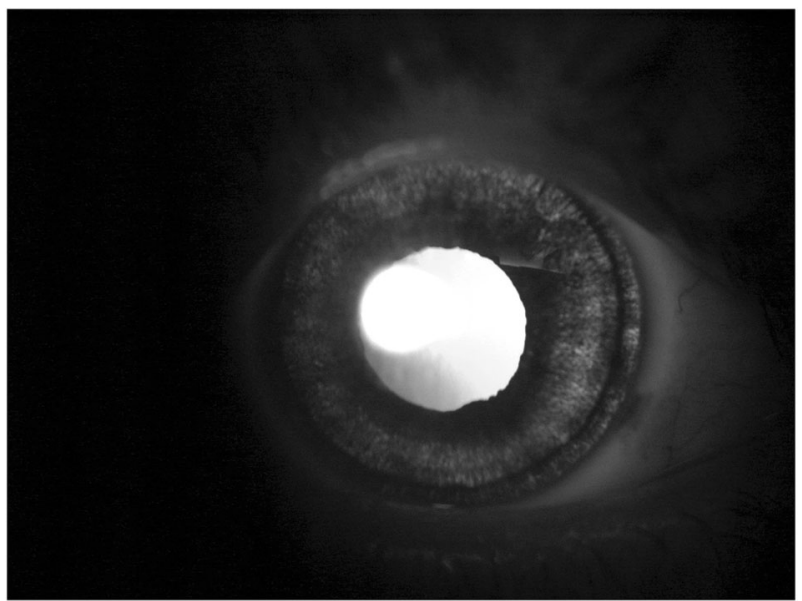

Fig. 1 Color photo of anterior segment demonstrating diffuse transillumination defects and fixed mid-dilated, irregular shaped pupil, 2 months following uptake of oral moxifloxacin

129 days (range: 93-167 days SD: 38 days). Table 1 summarizes the demographic and clinical characteristics of the patients.

Laboratory investigation was unremarkable or not conclusive in all patients and PCR results were negative for presence of herpetic viruses in the six cases tested. Mean baseline BCVA (SD) was $0.0181(0.5)$ with $4(12.5 \%)$ eyes experiencing reduction of vision. Visual loss recovered in all cases and mean BCVA (SD) improved to 0.0029 (0.112) at the final visit. All patients presented with severe bilateral photophobia, and conjunctival injection. Diffuse iris transilumination defects and mydriatic pupil with sphincter paralysis was a common finding in all 32 eyes, (Fig. 1) while an irregular shaped iris with distorted pupillary borders was documented in 20 eyes (63\%). Evidence of posterior synechiae was detected in 4 patients (8 eyes). On gonioscopy, angle was open but heavily pigmented in all eyes with no evidence of posterior iris bowing. The pigmentation of the trabecular meshwork was largely asymmetric, mainly involving the inferior quadrant.

Brisk anterior chamber activity, attributed to pigment dispersion rather than inflammatory cells, was detected in all patients. Pigment showering was severe during the first few weeks following onset of symptoms, declining over time. Application of the SUN criteria for the quantification of pigmented cells revealed mean (SD) baseline pigment in $\mathrm{AC}$ of 2.5 (0.9) improving to 0 (0) at the final visit. (Fig. 2)

Although, inflammatory keratic precipitates were not seen, there was evidence of fine pigment precipitates on the corneal endothelium in all eyes. The distribution of the precipitates involved the inferior cornea in 21 eyes; whereas, in 11 eyes pigment was diffusely scattered.

All 32 eyes were phakic and through-out the follow-up period posterior segment examination revealed no signs of vitritis, retinitis or choroiditis. Topical steroids under

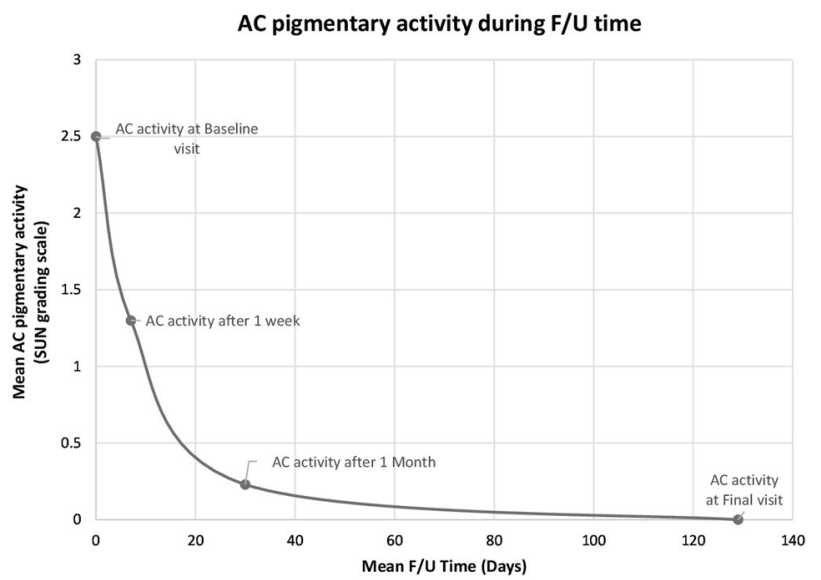

Fig. 2 Mean anterior chamber pigmentary activity during the followup period

variable regiments were administered in all cases and the mean duration (SD) of treatment was 4.0 (1.78) weeks.

Ocular Hypertension (OHT) was defined as IOP $>21$ $\mathrm{mmHg}$ and had a mean duration (SD) of 3.46 (3.3) weeks. Even though all 32 eyes developed OHT during the followup period, only thirty eyes had IOP $>21 \mathrm{mmHg}$ at the first visit. Mean IOP at baseline was $31 \pm 7 \mathrm{mmHg}$ (range: 16$41 \mathrm{mmHg}$ ) and 25 eyes required pressure lowering medications during the follow-up period. The maximum number of antiglaucoma agents used was 2.1 (1.5) and was reduced to $0.5(0.7)$ at the final visit.

Ocular hypertension persisted for at least 3 weeks in 15 eyes $(47 \%)$, despite aggressive antiglaucoma treatment. In one patient with intractable bilateral OHT AC washout of one eye was performed, in an attempt to reduce pigment in the AC and decongest the angle. Postoperative follow-up showed no notable difference as opposed to the fellow eye, regarding the AC activity and the course of OHT. Moreover, four eyes with persisting IOP $>40 \mathrm{mmHg}$ despite maximum antiglaucoma treatment, underwent filtration surgery which resulted in reduction of IOP and discontinuation of pressure lowering medication. Figure 3 summarizes the mean IOP variations during the follow-up.

Although older patients developed higher IOP at baseline $(p=0.022)$, final IOP was inversely correlated to patient age $(p<0.001)$. Higher IOP during the first week was associated with presence of posterior synechiae $(p=0.019)$, as well as a more refractory course of ocular hypertension, which required larger number of antiglaucoma agents at the end of the follow-up period. $(p=0.038)$.

Severe anterior chamber pigmentary activity (SUN grading: $3+$ or $4+$ ) in the 1 st week and the 1 st month was more likely to result in longer duration of OHT $(p=0.05$ and $p=0.019$, respectively) and in the administration of an increased number of antiglaucoma medications $(p<0.001$ and $p=0.021$, respectively). Finally, the presence of a 
Fig. 3 Mean IOP variations during the follow-up period. W = week, $\mathrm{M}=$ month

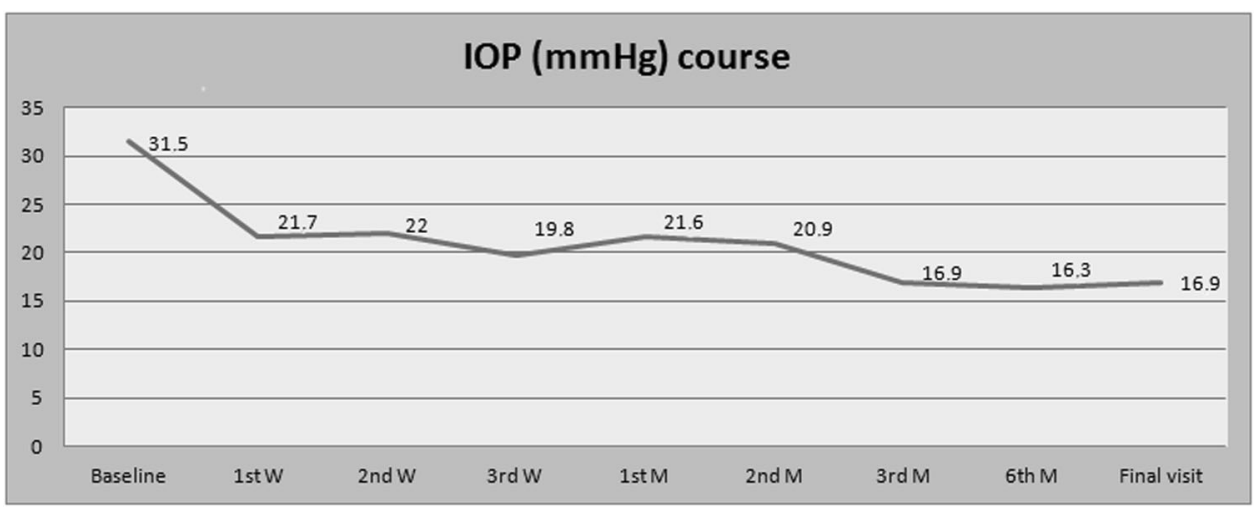

higher IOP during the first month of the follow-up period was also a strong prognostic factor for longer duration of ocular hypertension $(p<0.001)$.

\section{Discussion}

In this report, we described the demographic characteristics and the clinical course of 16 patients who developed severe photophobia with diffuse iris transillumination defects, mydriatic atonic pupils, and pigment dispersion in the anterior chamber, following administration of systemic antibiotics. The clinical entity of BAIT, which is characterized by the aforementioned signs has been reported to be associated with oral administration of moxifloxacin [611], and more rarely of other antibiotics, including clarithromycin and cefazoline $[12,13]$.

The association of BAIT with systemic use of antibiotics was challenged by Tugal-Tutkun et al. [1]. Although they have reported that $35 \%$ of their cases had previously been exposed to moxifloxacin treatment and $40 \%$ to other antibiotics, the authors speculated that a viral infection rather than moxifloxacin toxicity was more likely to be the causative factor of BAIT. Their conclusion was based on the lack-of ocular adverse effects after topical use of moxifloxacin, considering its effective ocular penetration.

In our series, 13 patients had previously been treated with systemic administration of moxifloxacin and three had been treated with clarithromycin. Although $50 \%$ of our patients had a history of pneumonia and flu like symptoms which could be attributable to viral etiology, this could not be the case for the rest of the cohort. Specifically, two of our cases received prophylactic antibiotic coverage after appendicectomy and nephrectomy and six were treated with systemic antibiotics after culture proven bacterial infection.

A standard dosage of antibiotics, according to each particular underlying disease, has been prescribed for every patient in this study; consequently, ocular signs and symptoms do not seem to be dosage dependent. Interestingly, 11 of 16 patients reported to be first-time users of systemic moxifloxacin or clarithromycin, suggesting a possible increased incidence rate of BAIT in these patients.

To date there have been no reports in the literature of ocular adverse effects on the iris epithelium following topical use of moxifloxacin. Moreover, toxicity studies have demonstrated a good safety profile of topical and intracameral use of moxifloxacin [14-16]. In addition, despite the high concentration of moxifloxacin following intravitreal administration, the rapid clearance of the drug within the first $12 \mathrm{~h}$ accounts for the lackof adverse reactions [17]. Conversely, oral uptake of moxifloxacin is associated with longer duration of the antibiotic within the ocular tissues explaining the adverse effects related to BAIT after systemic rather than intravitreal administration [18]. Knape et al. [7] raised the hypothesis that the lens status may be a contributing factor in BAIT. The authors suggested that trapping of the drug in the posterior chamber due to iridolenticular contact in phakic eyes may reduce its posterior to anterior clearance, resulting in higher drug concentrations which may be toxic for the iris epithelium.

Dispersion of pigment in the anterior chamber is a clinical feature of PDS. Iridolenticular contact due to posterior iris bowing has been considered to be the main causative factor of pigment dispersion in this setting, creating spokelike pattern mid peripheral transillumination defects and deposition of pigment on the corneal endothelium in the form of Krukenberg spindle [2]. Although AS-OCT was performed in only four of the patients in this study, (Fig. 4) no signs of posterior bowing of the iris have been observed during gonioscopy and slit-lamp biomicroscopy to the rest of the cohort. Moreover, the transilumination defects in our cases were both extensive and diffuse, involving a considerable proportion of the iris while there was no evidence of Krukenberg spindle in any of the eyes.

Pseudoexfoliation syndrome also represents a clinical entity characterized by transillumination defects. However, this condition tends to affect older population, is usually asymmetrical with transilumination defects involving the pupillary margin and fibrillary material deposition on the structures of the anterior segment. None of these features were present concerning the patients of this study. 


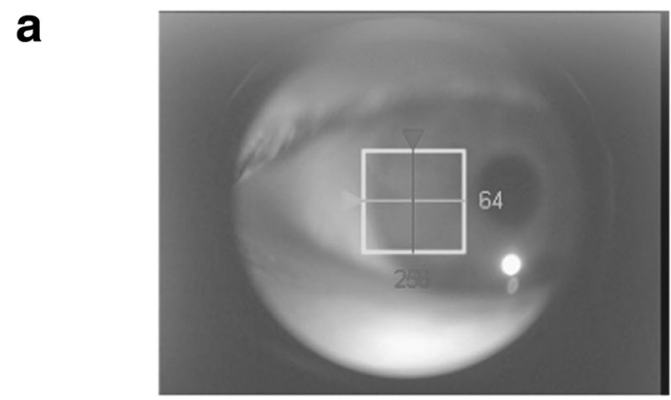

High-definition mode
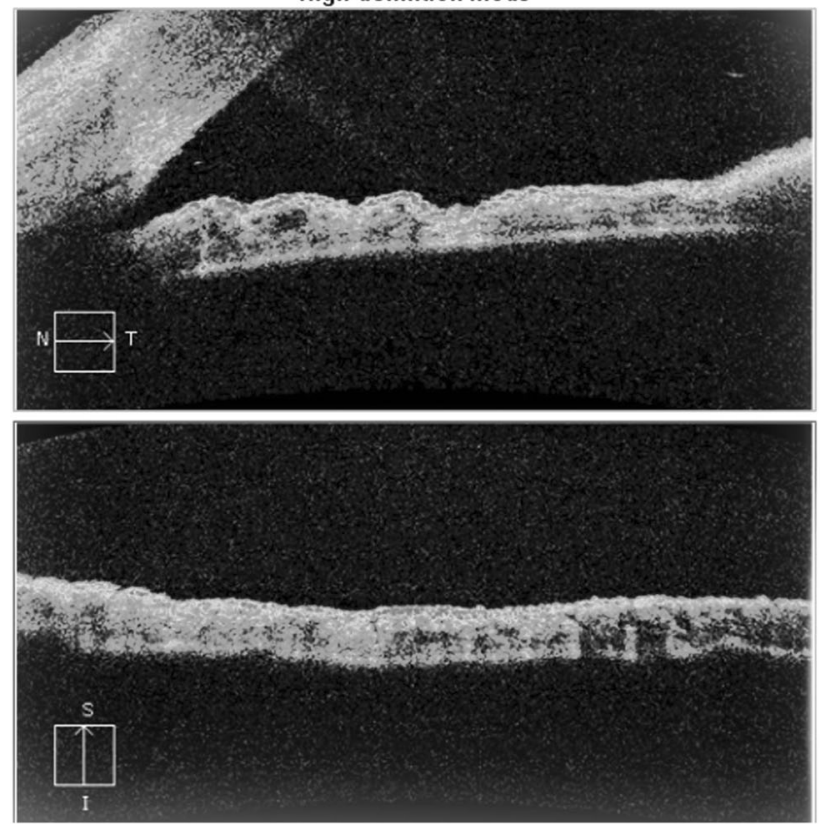

b

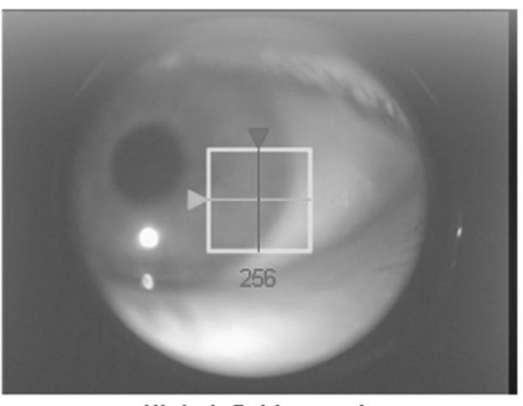

High-definition mode
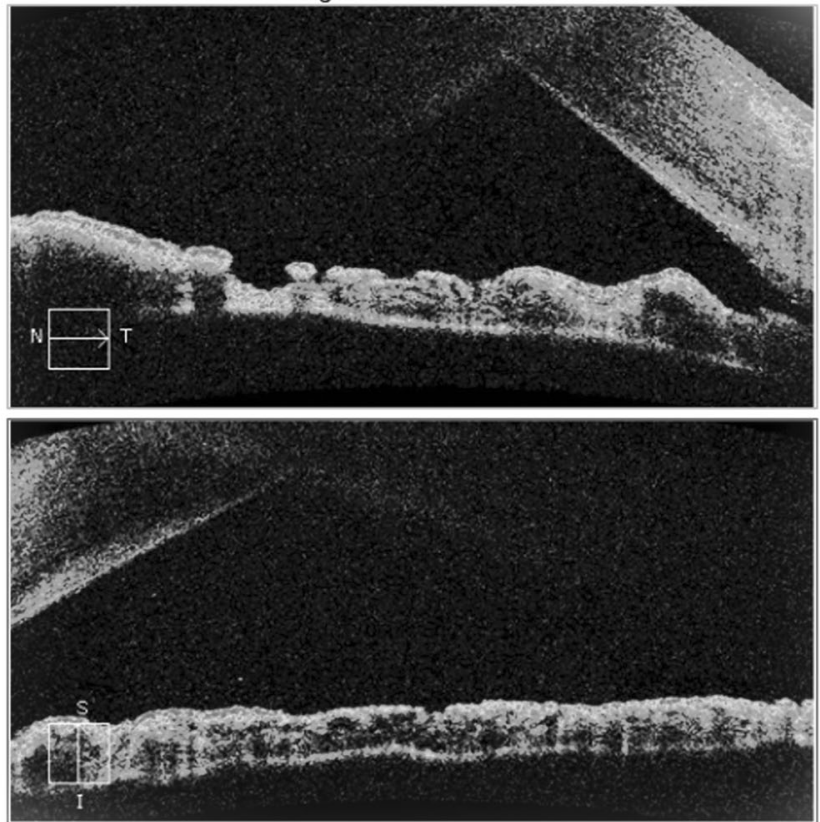

Fig. 4 Anterior segment OCT: (a) Right eye, (b) Left eye, no backbowing of the iris was observed

Bilateral acute depigmentation of the iris (BADI) which is a newly described clinical entity, also shares some initial symptoms with BAIT. However, in BADI it is the iris stroma that is predominantly affected and its depigmentation accounts for the pigment showering of the AC, while the iris pigment epithelium is the tissue most likely contributing to pigmentary dispersion in BAIT. Moreover, in BADI, eyes maintain intact pupillary reaction and there is a lack-of-transillumination defects [19].

Iris transillumination also occurs in viral Iridocyclitis and is thought to be the result of full thickness iris atrophy attributed, to an occlusive vasculitis or a direct viral invasion $[20,21]$. However, viral iridocyclitis is usually unilateral, sectoral, with intraocular inflammatory activity and frequent development of posterior synechiae. Even though posterior synechiae has been found in four eyes, no other clinical features associated with viral iridocyclitis were seen in our patients and PCR testing failed to reveal viral infection.

Our description of the demographic characteristics of patients with BAIT following the administration of oral moxifloxacin or Clarithromycin, confirm the female preponderance in the affected population described by other authors [1]. Moreover, a possible autoimmune predisposition in response to antibiotic intake may be the triggering factor for the characteristic signs encountered in this clinical entity [11]. Hinkle et al. reported 14 cases of uveitis associated with iris transillumination, atonic pupils, and pigment dispersion after systemic uptake of fluoroquinolones. The authors observed a female predisposition as well as a strong correlation with the HLA-B27 and HLA-51 haplotypes. HLA -B27 was found positive in two patients and HLA-51 in four patients of the 10 that had been tested. In our series, HLA testing was not performed in any of the patients; therefore, potential correlation between BAIT and these haplotypes cannot be determined.

Early rise of IOP is a well described feature of BAIT. In the present cohort, all patients developed ocular hypertension within the first 2 weeks after onset of symptoms, which persisted in $47 \%$ of the eyes for at least 3 weeks despite maximum antiglaucoma medication. In comparison, TugalTutkun et al. reported that $54 \%$ of their patients had OHT 
and two patients required trabeculectomy for intractable IOP. The exact etiology of OHT is not clear and it may be due to more than one contributing factors. In this study, a strong correlation between pigmentary AC activity and more refractory course of ocular hypertension has been observed. We speculate that the acute nature of the pigment showering results in remarkable congestion of the trabecular meshwork with consequent increase of the IOP.

Admittedly, the present study has some limitations that have to be mentioned. Namely, the retrospective nature of the study in combination with the limited follow-up period, the absence of iris angiography and the lack-of-AS-OCT in the majority of our patients are its most important limitations. Moreover, there is an inherent risk of recall bias in this retrospective study, particularly with five patients expressing their uncertainty in drug history. PCR analysis was performed only in six patients and there was no HLA testing. However, we have documented in a systematic way a relatively large and homogenous sample of eyes with BAIT, giving emphasis to the course of clinically significant ocular complications including ocular hypertension and pigment showering.

In conclusion, our study provides further clinical evidence on a rare adverse reaction of oral Moxifloxacin and Clarithromycin administration. Acute bilateral transillumination defects, pigment AC activity, and mid-dilated pupil are distinctive signs of this condition which is also accompanied by raised IOP in the majority of the cases. Physicians should be aware of this side effect and patients should be alert to the possibility of presenting the relative symptomatology during the first few weeks following oral administration of antibiotics. Even though all our patients have developed BAIT for the first time, it would be difficult to suggest any abstention from the same antibiotics in the future, due to lack-of additional data. Hence, future studies with longer follow-up period should be performed in order to address the aforementioned concern, as well as investigate the long-term effects of this clinical entity and to determine possible causative agents and pathogenic mechanisms.

\section{Summary}

\section{What was known before}

- BAIT is a condition characterized by acute bilateral iris transillumination and mid-dilated unresponsive pupils.

- Only a small amount of cases has been reported in the literature.

- The etiopathogenesis of this condition remains unclear.

\section{What this study adds}

- Our study indicates strong correlation between systemic antibiotic administration and BAIT.

- Further information about clinical features, complications, and management of this condition.

\section{Compliance with ethical standards}

Conflict of interest The authors declare that they have no conflict of interest.

\section{References}

1. Tugal-Tutkun I, Onal S, Garip A, Taskapili M, Kazokoglu H, Kadayifcilar S, et al. Bilateral acute iris transillumination. Arch Ophthalmol. 2011;129:1312-9.

2. Niyadurupola N, Broadway DC. Pigment dispersion syndrome and pigmentary glaucoma - a major review. Clin Exp Ophthalmol. 2008;36:868-82.

3. Mohamed Q, Zamir E. Update on Fuchs' uveitis syndrome. Curr Opin Ophthalmol. 2005;16:356-63.

4. Markomichelakis NN, Canakis C, Zafirakis P, Marakis T, Mallias I, Theodossiadis G. Cytomegalovirus as a cause of anterior uveitis with sectoral iris atrophy. Ophthalmology. 2002;109:879-82.

5. Suhler EB, Buggage RR, Nussenblatt RB, Neumann R. Symmetric peripheral iris depigmentation in vogt-koyanagi-harada syndrome. Arch Ophthalmol. 2002;120:1104-5.

6. Morshedi RG, Bettis DI, Moshirfar M, Vitale AT. Bilateral acute iris transillumination following systemic moxifloxacin for respiratory illness: report of two cases and review of the literature. Ocul Immunol Inflamm. 2012;20:266-72.

7. Knape RM, Sayyad FE, Davis JL. Moxifloxacin and bilateral acute iris transillumination [letter]. J Ophthalmic Inflamm Infect. 2013;3:10.

8. Willermain F, Deflorenne C, Bouffioux C, Janssens X, Koch P, Caspers L. Uveitis-like syndrome and iris transillumination after the use of oral moxifloxacin. Eye. 2010;24:1419. [author reply] 1419-20

9. Wefers Bettink-Remeijer M, Brouwers K, van Langenhove L, De Waard PW, Missotten TO, Martinez Ciriano JP, Van Aken E. Uveitis-like syndrome and iris transillumination after the use of oral moxifloxacin. Eye. 2009;23:2260-2.

10. Nascimento HM, Sousa JM, Campos MS, Belfort R Jr.. Acute iris depigmentation following systemic moxifloxacin. Clinics. 2013;68:899-900.

11. Hinkle DM, Dacey MS, Mandelcorn E, Kalyani P, Mauro J, Bates $\mathrm{JH}$, et al. Bilateral uveitis associated with fluoroquinolone therapy. Cutan Ocul Toxicol. 2012;31:111-6.

12. Tranos P, Nasr MB, Asteriades S, Vakalis A, Georgalas I. Bilateral diffuse iris atrophy after the use of oral clarithromycin. Cutan Ocul Toxicol. 2014;33:79-81.

13. Gonul S, Bozkurt B. Bilateral acute iris transillumination (BAIT) initially misdiagnosed as acute iridocyclitis. Arq Bras Oftalmol. 2015;78:115-7.

14. Lane SS, Osher RH, Masket S, Belani S. Evaluation of the safety of prophylactic intracameral moxifloxacin in cataract surgery. $\mathrm{J}$ Cataract Refract Surg. 2008;34:1451-9.

15. Kernt M, Hirneiss C, Neubauer AS, Liegl RG, Eibl KH, Wolf A, et al. Intracameral moxifloxacin: a safe option for endophthalmitis 
prophylaxis? In vitro safety profile for intraocular application. Ophthalmologe. 2010;107:720-7.

16. Zhou AX, Messenger WB, Sargent S, Ambati BK. Safety of undiluted intracameral moxifloxacin without postoperative topical antibiotics in cataract surgery. Int Ophthalmol. 2016;36:493-8.

17. Iyer MN, He F, Wensel TG, Mieler WF, Benz MS, Holz ER. Intravitreal clearance of moxifloxacin. Trans Am Ophthalmol Soc. 2005;103:76-83.

18. Fukuda M, Shibata N, Osada H, Yamashiro Y, Sasaki H. Vitreous and aqueous penetration of orally and topically administered moxifloxacin. Ophthalmic Res. 2011;46:113-7.
19. Tugal-Tutkun I, Urgancioglu M. Bilateral acute depigmentation of the iris. Graefes Arch Clin Exp Ophthalmol. 2006;244:742-6.

20. Liesegang TJ. Varicella-zoster virus eye disease. Cornea. 1999;18:511-31.

21. Liesegang TJ. Classification of herpes simplex virus keratitis and anterior uveitis. Cornea. 1999;18:127-43.

22. Butler NJ, Suhler EB. Levofloxacin-associated panuveitis with chorioretinal lesions. Arch Ophthalmol. 2012;130:1342-4.

23. Brooks AM, West RH, Gillies WE. Acute primary ischemic iris atrophy. Ophthalmology. 1988;95:1234-43. 